\title{
Article
}

\section{Improved Analytical Theory of Ophthalmic Lens Design}

\author{
Eduardo Pascual ${ }^{1, *(\mathbb{D}}$, José A. Gómez-Pedrero ${ }^{2}$ and José Alonso ${ }^{1}$ \\ 1 Indizen Optical Technologies S.L. Suero de Quiñones 34, 28002 Madrid, Spain; jalonso@iot.es \\ 2 Applied Optics Complutense Group, Facultad de Óptica y Optometria, Universidad Complutense de Madrid, \\ Arcos de Jalón, 118, 28037 Madrid, Spain; jagomezp@fis.ucm.es \\ * Correspondence: epascual@iot.es; Tel.: +34-91-833-37-86
}

check for updates

Citation: Pascual, E.;

Gómez-Pedrero, J.A.; Alonso, J. Improved Analytical Theory of Ophthalmic Lens Design. Appl. Sci. 2021, 11, 5696. https://doi.org/ 10.3390/app11125696

Academic Editor: José Antonio Díaz Navas

Received: 31 May 2021

Accepted: 17 June 2021

Published: 19 June 2021

Publisher's Note: MDPI stays neutral with regard to jurisdictional claims in published maps and institutional affiliations.

Copyright: (c) 2021 by the authors. Licensee MDPI, Basel, Switzerland. This article is an open access article distributed under the terms and conditions of the Creative Commons Attribution (CC BY) license (https:/ / creativecommons.org/licenses/by/ $4.0 /)$.
Featured Application: Analytical tools for ophthalmic lens design that may guide numerical optimization.

Abstract: A revisited form of the classic third-order ophthalmic lens design theory that provides a more precise and meaningful use of aspheric surfaces and a generalization of the standard oblique errors is presented. The classical third-order theory follows from the application of the Coddington equations to a ray trace through the lens and the expansion of the incidence angles and the surface sagittas appearing on them up to order two of the radial coordinate. In this work we show that the approximations for surface sagittas and angles can be decoupled, and the lens oblique powers predicted by the proposed theory provides a better fit to the numerical results obtained by exact raytracing and multi-parametric optimization than the classical third-order theory does. Modern ophthalmic lens design uses numerical optimization and exact ray tracing, but the methods presented in this paper provide a deeper understanding of the problem and its limitations. This knowledge and the more general merit functions that are also presented may help guide the numerical approaches.

Keywords: ophthalmic lenses; Coddington equations; optimization; exact ray tracing

\section{Introduction}

Spectacle lenses can be designed considering several factors, such as visual performance, aesthetics (thickness or curvature of the front surface, also known as base curve), or manufacturing limitations. When visual performance is considered, the usual strategies are primarily focused on the minimization of oblique aberrations, which are the residual defocus and astigmatism appearing at oblique gaze directions or when the lenses are tilted. Secondary objectives can be the reduction of distortion, higher-order aberrations, or even chromatic aberrations [1-10].

The first complete approaches to the management of oblique aberrations were based on the so-called third-order approximation [6,9], although trigonometric ray tracing was also used early on in the development of obliquely corrected eyeglasses [11]. Nowadays, computers allow us the construction of elaborate lens-eye models that can be explored with detail by means of exact ray tracing or wavefront tracing $[5,12,13]$. Lens performance can be analyzed in terms of the number of oblique errors or the size of the geometrical blur patch on the retina $[6,14]$. The computation of geometrical wavefronts and the corresponding coefficients for higher-order aberrations or optical transfer functions have led many authors to conclude that ophthalmic lens performance is indeed mainly determined by oblique errors [15].

Although modern lens design may involve exact ray tracing and recurrence or optimization algorithms [16,17], the third-order approach is still useful for getting an initial point for more complex analysis, or to find key relationships and limitations that would guide further design. The classical procedure for obtaining analytical expressions of the oblique errors in lenses with revolution symmetry is based on the use of Coddington's equations, which provide the location of the tangential and sagittal foci for a given surface. 
Repeated application of these equations to the two lens surfaces, along with the use of third-order and thin-lens approximations to compute incidence and refracted angles, leads to analytical expressions for tangential and sagittal lens vergences or powers. The main and classical result from this analysis, when applied to spherical lenses, are the well-known Tscherning ellipses [6]. They provide the combination of base curve and prescription for which either oblique astigmatism, mean power error, or tangential error are cancelled. In general, the third-order solution takes the form

$$
E=x^{2} f_{s p h}\left(\kappa_{i}, P, n, L\right)
$$

where $E$ stands for some type of oblique error (classically, oblique astigmatism, oblique mean power error, or tangential error), $x$ is the radial coordinate, or distance from the optical axis, of the point the viewing line is passing through, and $f_{\text {sph }}$ is a quadratic function of the curvature of either surface, $\kappa_{1}$ or $\kappa_{2}$, and the lens power, $P$; it also depends on the lens refractive index, $n$, and the object vergence, $L$. More generally, $f_{s p h}$ will be a function of the curvature of both surfaces and the lens power. For thin lenses, they are related by the lens equation, so only two of them are needed to specify lens power and lens bending. In modern lens design, the front surface is usually spherical, and its refractive power is known as the base curve, $B$. Setting $f_{\text {sph }}(B, P)=0$ leads to the Tscherning ellipse for the particular oblique error, and it establishes a relationship between lens power and base curve that will cancel the error. With $f_{\text {sph }}(B, P)=0$ being a closed curve, there is a finite range of powers for which the error cancelation can be achieved. Additionally, for any particular power within this range, there will be two values (Ostwald's and Wollaston's solutions) of the base curve that will seat on the ellipse, henceforth cancelling the oblique error. Because of the form of the previous equation, cancellation will take place for all values of $x$; that is, third-order theory applied to spherical lenses predicts that a given oblique error will be cancelled at full field if the base curve is selected so that $f_{\text {sph }}(B, P)=0$. Finally, as each oblique error has its own function $f_{\text {sph }}(B, P)$, only one type of error can be cancelled.

Lenses with aspherical surfaces but still keeping revolution symmetry have also been thoroughly studied. The extension of the third-order theory to include lenses with aspherical surfaces has usually been based on a temporary fourth-order approximation to conicoids $[2,8,18]$. This procedure provides one extra parameter per aspheric surface: the conic constant. Once the asphericity is incorporated into the equations, a new second-order approximation on the transverse coordinate $x$ yields a result similar to that of spherical lenses,

$$
E=x^{2} f_{\text {asph }}\left(B, P, Q_{1}, Q_{2}, n, L\right)
$$

where $Q_{1}$ and $Q_{2}$ are, respectively, the conic constants of the front and back lens surfaces, and $f_{\text {asph }}$ is no longer a quadratic equation on $B$ and $P$. The extra degrees of freedom provided by the asphericity allows for extending the range of lens powers for which there is a solution $f_{a s p h}=0$, which is infinite now. Moreover, a solution $f_{a s p h}=0$ can be obtained for any base curve, provided we select the conic constants adequately. Finally, the fact that $f_{a s p h}$ does not depend on $x$ implies that correction of the oblique error is uniform across the whole field. However, when aspheric lenses with conicoid surfaces are studied by means of exact ray tracing, it is observed that full field compensation of the oblique error cannot be achieved $[9,17]$. Numerical computations reveal that, indeed, any lens power can be corrected of one oblique error for any value of the base curve, but correction takes place at a single viewing direction (a single value of the $\mathrm{x}$ coordinate) and then, typically, performance rapidly deteriorates as the viewing angle increases [9]. The reason for this disparity between the two computation approaches is that although local curvatures of the conicoids are incorporated into the Coddington equations through a fourth-order approximation (which is the bare minimum to introduce the conic constants), the resulting equations for the oblique errors are finally simplified by just retaining up to second-order terms in $x$. 
One important conclusion that can be deduced from the third-order approach is that, assuming one surface of the lens is kept spherical, it is not possible to simultaneously correct the astigmatism error and mean power error. That means all spectacle lenses (with at least one spherical surface) will have some uncorrected errors that might affect the visual quality. Therefore, a compromise is needed in how the unwanted power error is distributed between the mean power and the astigmatism. A usual strategy involves the definition of a merit function that can include terms for the oblique astigmatism, lens thickness, distortion, or higher-order aberrations. One example of such merit function is the LeGrand criterion described by Texier et al. [12], which uses a weight of $\sqrt{2} / 2$ on the astigmatism relative to the mean power. Another example could be the minimization of the blur as defined by Raasch [19], which implies a relative weight of $1 / 2$ on the astigmatism relative to the mean power. Sun et al. [1] proposed a merit function that combines oblique astigmatism, distortion, and a restriction to avoid inflection points on the surface.

As we have previously described, third-order theory has been applied to demonstrate the existence of solutions for merit functions that correct either oblique astigmatism, mean power error, or tangential error, but the question about the existence of solutions for general merit functions is still unanswered. Miks et al. [8] described results for aspherical lenses that eliminates oblique astigmatism while reducing high-order aberrations. Texier et al. [12] showed the result for the LeGrand criterion for both spherical and aspherical lenses.

The objective of this paper is twofold: first we show that third-order theory can be extended by keeping a more accurate description of the surface curvatures as required in the Coddington equations. Then, we use the resulting expressions for the tangential and sagittal powers to obtain a general solution beyond the particular cases aimed at the minimization of either oblique astigmatism, tangential error, or mean power error.

This paper focuses on ophthalmic single-vision lenses. Moreover, the powers considered will be moderate, below five diopters, as they represent the refractive errors of the majority of the lens wearers [20]. Therefore, we can assume that both surfaces in the lens have low curvature. Considering the de facto standard in the modern ophthalmic industry, we will assume that the front surface is spherical with refractive power $B$ and that the back surface is aspherical, and we will study the properties of the solution with respect to the asphericity and the base curve. More complex lens geometries such as bi-aspheric lenses have been designed and commercialized. When both surfaces are free, the tangential and sagittal errors can be independently optimized $[17,21]$. The results presented in this paper can be extended to bi-aspherical lenses, but we restrict the analysis to lenses with one spherical surface. Finally, we demonstrate that, within this extended third-order theory, we have one and only one degree of freedom to cancel any arbitrary combination of oblique errors. Extensive numerical experiments using exact ray tracing confirm that these results are not a consequence of the approximations but are an intrinsic feature of ophthalmic lens geometry and use.

\section{Method}

The first part of the analysis follows the standard procedure to locate tangential and sagittal foci of an ophthalmic lens. Though the reader may find this computation elsewhere $[3,6]$, we have opted to reproduce the standard development here for completeness and to make clearer the different approximations we later use. First, we consider the refraction of a thin astigmatic pencil whose principal ray lays in a meridional plane of a surface with revolution symmetry (that is, the symmetry axis and the main ray are co-planar). Let $n$ and $n^{\prime}$ be the refractive indexes at either side of the surface, as depicted in Figure 1 . The tangential and sagittal object points are located at points $Q_{T}$ and $Q_{S}$. After the principal ray is refracted at point $G$, the tangential image for $Q_{T}$ is $Q_{T}^{\prime}$, and the sagittal image point for $Q_{S}$ is $Q_{S}^{\prime}$. The vector $\mathbf{N}$ is the normal to the surface $S$ at point $G$. 


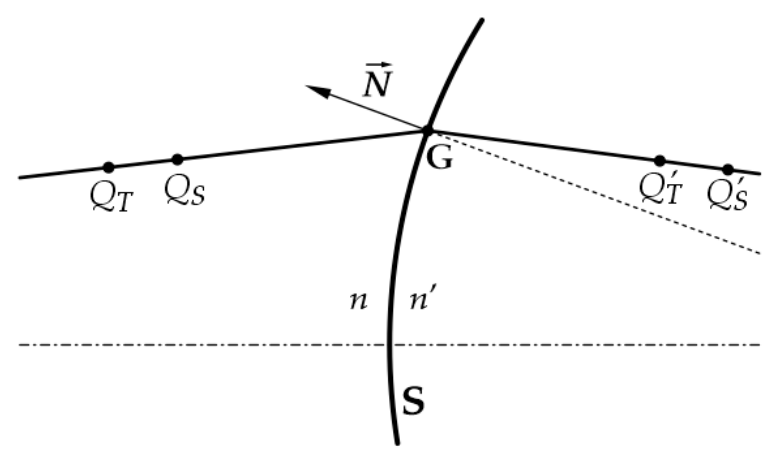

Figure 1. Ray refracting through a surface S.

The relationship between the object and image points are given by the Coddington equations

$$
\begin{gathered}
\frac{n^{\prime}}{s^{\prime}}-\frac{n}{s}=\frac{n^{\prime} \cos a-n \cos b}{R_{s}}, \\
\frac{n^{\prime} \cos ^{2} a}{t^{\prime}}-\frac{n \cos ^{2} b}{t}=\frac{n^{\prime} \cos a-n \cos b}{R_{t}},
\end{gathered}
$$

where $s=\overline{Q_{S} \mathrm{G}}, t=\overline{Q_{T} \mathrm{G}}, s^{\prime}=\overline{\mathrm{GQ}_{S}^{\prime}}$, and $t^{\prime}=\overline{\mathrm{GQ}_{T}^{\prime}}$. $R_{S}$ and $R_{T}$ are the main radii of curvature at $\mathrm{G}$ (sagittal and tangential, respectively). The angles $a$ and $b$ are the incidence and refraction angles of the principal ray at $G$.

Let us now consider a lens of refractive index $n$ surrounded by air, as shown in Figure 2. Let the object point be located at $\mathrm{Q}$, so $t_{1}=s_{1}=\overline{\mathrm{GQ}}$. Consider a thin ray pencil, which hits point $G$ on the front surface, $S_{1}$. The incidence and refracting angles of its principal ray are called $a$ and $b$, respectively. The ray pencil propagates inside the lens up to a point $\mathrm{H}$ on the second surface, striking this surface with an incidence angle $c$ and emerging with refraction angle $d$. In general, the emerging ray pencil is astigmatic, being $t_{2}^{\prime}=\overline{\mathrm{HQ}_{T}^{\prime}}$ and $s_{2}^{\prime}=\overline{\mathrm{HQ}_{S}^{\prime}}$.

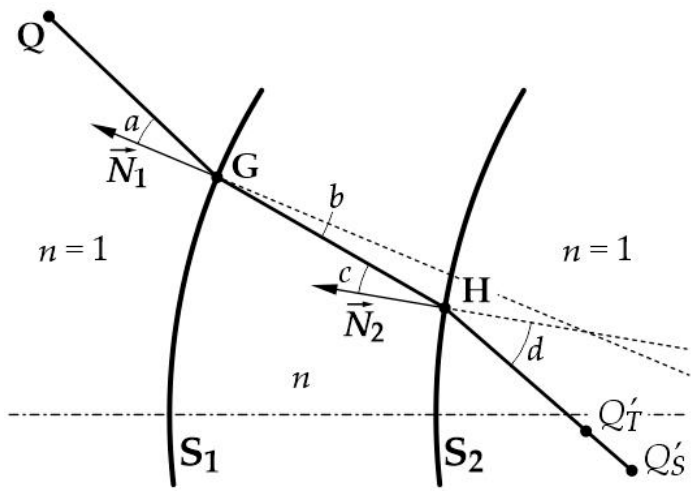

Figure 2. Ray propagation through a lens.

To obtain the formulas for the sagittal and tangential powers, the Coddington equations are applied to the points $\mathrm{G}$ and $\mathrm{H}$. For the first surface the equations are

$$
\begin{gathered}
\frac{n}{s_{1}^{\prime}}-\frac{1}{s_{1}}=\frac{n \cos b-\cos a}{R_{1 S}} \\
\frac{n \cos ^{2} b}{t_{1}^{\prime}}-\frac{\cos ^{2} a}{t_{1}}=\frac{n \cos b-\cos a}{R_{1 T}}
\end{gathered}
$$

and for the second surface

$$
\frac{1}{s_{2}}-\frac{n}{s_{2}}=\frac{\cos d-n \cos c}{R_{2 S}}
$$




$$
\frac{\cos ^{2} d}{t_{2}^{\prime}}-\frac{n \cos ^{2} c}{t_{2}}=\frac{\cos d-n \cos c}{R_{2 T}} .
$$

$R_{1 S}$ and $R_{2 S}$ are the local curvature radii in the sagittal plane of the first and second surfaces at points $\mathrm{G}$ and $\mathrm{H}$, respectively. The same convention applies for the radii $R_{1 T}$ and $R_{2 T}$. Next, we will calculate the oblique powers using Equations (3)-(6) to calculate the oblique powers, following the procedure used by Jalie [6]. If the distance $\overline{\mathrm{GH}}$ can be neglected with respect to the surface radii and focal distances, then

$$
t_{1}^{\prime} \cong t_{2}, s_{1}^{\prime} \cong s_{2}
$$

In addition, according to Snell's law

$$
\sin a=n \sin b, \quad n \sin c=\sin d .
$$

In classical third-order theory, the angles are supposed to be small, so the second-order approximation can be used:

$$
\sin a \cong a, \quad \cos a \cong 1-a^{2} / 2 .
$$

On the other hand, the local radii can be replaced by the surface refractive powers, $F_{1 S, T}=B=(n-1) / R_{1 S, T}$ and $F_{2 S, T}=-(n-1) / R_{2 S, T}$ Finally, the lens sagittal and tangential powers are defined as $F_{S}=1 / s_{2}^{\prime}$ and $F_{T}=1 / t_{2}^{\prime}$.

Substituting the previous definitions and relations into the Coddington equations, we can obtain expressions for the oblique powers that are linear on the surface refractive powers and quadratic on the angles $a$ and $d$

$$
\begin{gathered}
F_{S}=\left(B+F_{2 S}\right)\left(1+\frac{a^{2}}{2 n}\right)-F_{2 S} \frac{a^{2}-d^{2}}{2 n}, \\
F_{T}=\left(B+F_{2 T}\right)\left[1+\frac{(n+2) a^{2}}{2 n^{2}}+\frac{\left(n^{2}-1\right) d^{2}}{n^{2}}\right]-\frac{F_{2 T}(n+2)\left(a^{2}-d^{2}\right)}{2 n^{2}} .
\end{gathered}
$$

The angle $d$ can be determined by the position of the lens relative to the eye, as shown in Figure 3. As it is usually done in ophthalmic lens design, we are assuming that the eye is looking through point $\mathrm{H}$ and that its visual axis coincides with the refracted principal ray. This also means the rotation center of the eye is located at $\mathrm{O}_{2}^{\prime}$, where the output ray meets the lens optical axis. We also use the classical assumption that the aperture stop and the exit pupil are located at the rotation center of the eye. When the eye rotates an angle $\theta_{2}^{\prime}$, its visual axis goes through point $\mathrm{H}$, whose transverse or radial coordinate with respect to the optical axis is $x$. The distance from the vertex of the back surface to $\mathrm{O}_{2}^{\prime}$ (also the rotation center of the eye) is $l_{2}^{\prime}$, with a related vergence $L_{2}^{\prime}$.

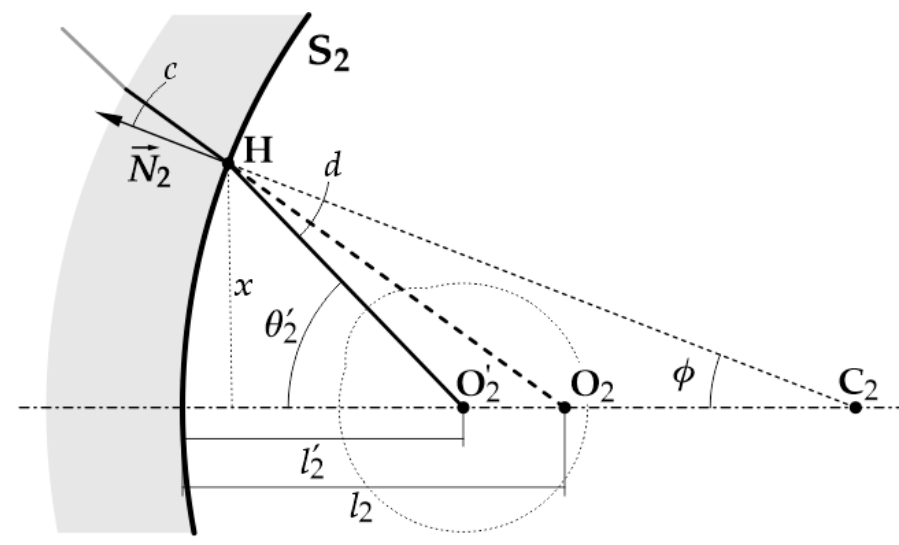

Figure 3. Parameters describing the refraction at the back surface of the lens. 
The angle $d$ is given by

$$
d=\phi-\theta_{2}^{\prime}
$$

where $\phi$ is the angle formed between $N_{2}$ and the optical axis. If the transverse coordinate $x$ is small with respect to the radius of curvature of the surface at its vertex, we can approximate

$$
\phi(x) \cong-\frac{x F_{2}(0)}{n-1}, \quad \theta_{2}^{\prime}=x L_{2}^{\prime}
$$

As the lens is rotationally symmetric and we are neglecting its thickness, its central power is given by $P=B+F_{2}(0)$. The angle $d$ is finally given by the expression

$$
d=-x \frac{P-B+L_{2}^{\prime}(n-1)}{n-1} .
$$

A similar analysis can be made for the incidence angle $a$ at the first surface, as depicted in Figure 4. First, we have

$$
a=\varphi-\theta_{1}
$$

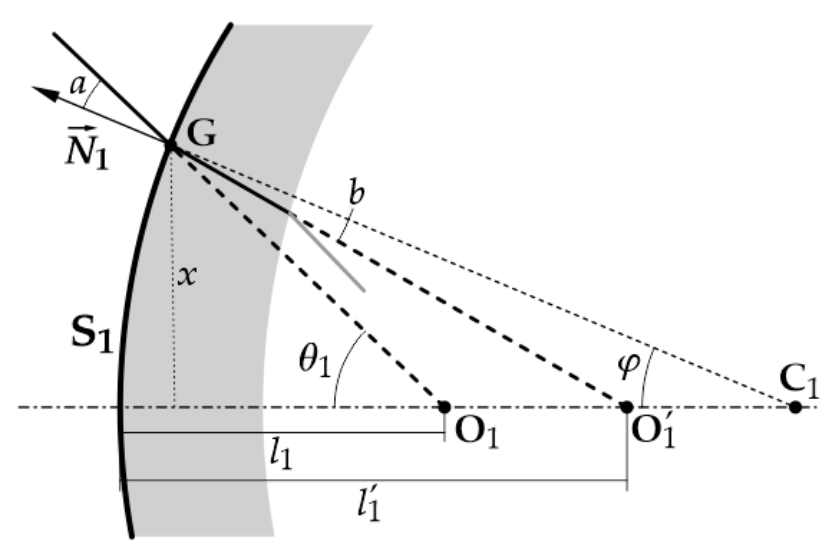

Figure 4. Parameters describing the refraction at the front surface of the lens.

With the same approximations we used for $d$, under which the transverse coordinate of $\mathrm{G}$ is also $x$, we have

$$
\theta_{1} \cong x L_{1}, \varphi(x) \cong \frac{x B}{n-1},
$$

where we are assuming that the transverse coordinates at $\mathrm{G}$ and $\mathrm{H}$ are the same, as a consequence of the thickness being negligible. Likewise, if we call $\mathrm{O}_{1}$ and $\mathrm{O}_{1}^{\prime}$ to the points where the incident and refracted rays cross the optical axis, $l_{1}$ and $l_{1}^{\prime}$ to the distances from the surface vertex to them, and $L_{1}$ and $L_{1}^{\prime}$ to their respective vergences, we can set $L_{1}=L_{1}^{\prime}-B, L_{1}^{\prime} \cong L_{2}$ and $L_{2}^{\prime} \cong L_{2}-(P-B)$. With all these equations we get the next expression:

$$
a=x \frac{\left[n P-(P-B)-L_{2}^{\prime}(n-1)\right]}{n-1} .
$$

Now we can substitute the expression for the angles (14) and (17) into the equations for the oblique power, (10) and (11). The final equations are quadratic in $x$, 


$$
\begin{gathered}
F_{T}(x)=\left[B+F_{2 T}(x)\right]\left\{\frac{x^{2}(n+2)\left[B-P+P n-L_{2}^{\prime}(n-1)\right]^{2}}{2 n^{2}(n-1)^{2}}\right. \\
\left.+\frac{x^{2}\left(n^{2}-1\right)\left[P-B+L_{2}^{\prime}(n-1)\right]^{2}}{n^{2}(n-1)^{2}}+1\right\} \\
-\frac{1}{2 n^{2}}\left\{\frac{x^{2}\left[P-B+L_{2}^{\prime}(n-1)\right]^{2}}{(n-1)^{2}}\right. \\
\left.-\frac{x^{2}\left[B-P+P n-L_{2}^{\prime}(n-1)\right]^{2}}{(n-1)^{2}}\right\}(n+2) F_{2 T}(x), \\
F_{S}(x)=\left[B+F_{2 S}(x)\right]\left\{\frac{x^{2}\left[B-P+P n-L_{2}^{\prime}(n-1)\right]^{2}}{2 n(n-1)^{2}}+1\right\} \\
+\frac{F_{2 S}(x)}{2 n}\left\{\frac{x 2\left[P-B+L_{2}^{\prime}(n-1)\right]^{2}}{(n-1)^{2}}\right. \\
\left.-\frac{x^{2}\left[B-P+P n-L_{2}^{\prime}(n-1)\right]^{2}}{(n-1)^{2}}\right\} .
\end{gathered}
$$

Note that in the last two equations we have made explicit the dependence of the sagittal and tangential powers on $x$. The differences between the tangential and sagittal powers and the central powers are known as tangential and sagittal errors, respectively: $E_{T}(x)=$ $F_{T}(x)-P, E_{S}(x)=F_{S}(x)-P$. Similarly, the oblique astigmatism and oblique mean power errors are defined as $E_{A}(x)=F_{T}(x)-F_{S}(x)$ and $E_{P}(x)=\left[F_{T}(x)+F_{S}(x)\right] / 2-P$.

\section{Results}

We continue to use $x$ as the transverse (radial) coordinate, and we give the name $z(x)$ to the sag of the back surface. For surfaces of revolution with low curvature, the local tangential and sagittal radii can be obtained from the simple relations $R_{T}(x)=1 / z^{\prime \prime}(x)$ and $R_{S}=x / z^{\prime}(x)$ [22]. Henceforth, the corresponding refractive powers are

$$
\begin{aligned}
& F_{2 T}(x)=-(n-1) z^{\prime \prime}(x), \\
& F_{2 S}(x)=-(n-1) \frac{z^{\prime}(x)}{x} .
\end{aligned}
$$

In principle, given any aspherical back surface with revolution symmetry, $z(x)$, we can compute its local refractive powers and then substitute in Equations (18) and (19) to obtain an approximation of the oblique powers of the lens. Then we would seek the conditions for which $E_{T}(x)=E_{S}(x)=0$, that is, there are no oblique aberrations; the lens wearer perceives the same power for any oblique sight direction. In general, this cannot be achieved, and some classical compromises have been proposed and thoroughly studied [6]:

- $\quad$ Point focal lens. Imposes the oblique astigmatism to be zero for all $x, E_{A}(x)=0$.

- Percival lens. Imposes the mean power to be equal to the central power for all $x$ : $E_{P}(x)=0$.

- Zero-tangential-error lens. Imposes the tangential power to be equal to the central power for all $x: E_{T}(x)=0$.

Instead of investigating each of these compromises separately, we may impose that a weighted average of the oblique (tangential and sagittal) powers must equal the central power,

$$
v F_{T}+u F_{S}=(u+v) P,
$$

where the weights $u$ and $v$ can take negative values. Any two pairs $(u, v)$ and $\left(u^{\prime}, v^{\prime}\right)$ lead to the same solution if they are linearly dependent, so condition (22) is fully determined by a single parameter, and a relation between $u$ and $v$ must be chosen. One such relation that will allow the generation of all interesting balances between tangential, sagittal, and central power while keeping a finite range of the parameters $u$ and $v$ is 


$$
u^{2}+v^{2}=1,
$$

which normalizes the 2-tupla $(u, v)$. Condition (22), along with normalization (23), can also be written as a balanced elimination of both the tangential and sagittal errors,

$$
E_{T} \cos \alpha+E_{S} \sin \alpha=0,
$$

where $v=\cos \alpha$ and $\alpha$ runs from $-\pi$ to $\pi$ to generate all the interesting cases.

The solutions for condition (22) includes the classical ones:

- Point focal lens, $u=-v=-\sqrt{2} / 2$, and $\alpha=-\pi / 4$.

- Percival lens, $u=v=\sqrt{2} / 2$, and $\alpha=\pi / 4$.

- Lens with zero tangential error, $u=0, v=1$, and $\alpha=0$.

Although not considered a classical solution, the lens with zero sagittal error can also be obtained when $u=1$ and $v=0(\alpha=\pi / 2)$.

In the traditional third-order theory, suitable functions describing $F_{2 S}(x)$ and $F_{2 T}(x)$ are substituted in Equations (18) and (19); the resulting expressions are expanded in powers of $x$, and the results are simplified by retaining up to second-order terms in $x$. This procedure leads to the generic expressions $E=x^{2} f_{\text {asph }}\left(B, P, Q_{1}, Q_{2}, n, L\right)$ we previously introduced. With this approach, condition (22) only adds marginal generality, as the dependence of any combination of oblique error will always be quadratic with $x$, and will vanish for all $x$ if $f_{\text {asph }}$ can be zeroed.

We show next that analytical results around condition (22) can be easily obtained without the need to neglect higher powers of $x$ in Equations (18) and (19). With the outgoing results we are able to explore how (22) is satisfied as a function of the transverse coordinate, and that brings a richer understanding of the full-field lens optimization problem. Finally, we will later see that among the family of oblique powers that satisfy condition (22), we can find the solution to the minimization of more interesting merit functions-for example, those related to optical blur averaged across the field of view.

When expressions (20)-(22) are applied to Equations (18) and (19), the problem is converted into a second-order linear ordinary differential equation, for which finding an explicit solution is not generally possible. However, if the surface has a Taylor series representation, $z(x)=\sum_{i>0 \text { even }} c_{i} x^{i}$, it can be inserted into Equations (20) and (21), and the uniqueness of the Taylor series yields similar expansions for the surface powers $F_{2 T}$ and $F_{2 S}$,

$$
\begin{gathered}
F_{2 T}(x)=-(n-1) \sum_{i \geq 0 \text { even }}(i+2)(i+1) c_{i+2} x^{i} \\
F_{2 S}(x)=-(n-1) \sum_{i \geq 0 \text { even }}(i+2) c_{i+2} x^{i} .
\end{gathered}
$$

Now, using these expansions in Equations (18) and (19) delivers the oblique powers also as a power series in $x$. Finally, we can use them in condition (22) and solve for the coefficients $c_{i}$ that fulfill the condition. The first two coefficients are

$$
\begin{gathered}
c_{2}=\frac{B-P}{2(n-1)}, \\
c_{4}=\frac{P}{8 n(u+3 v)(n-1)^{3}} \Delta,
\end{gathered}
$$

where $\Delta$ is a shorthand description for the quantity

$$
\begin{aligned}
\Delta=B^{2}[u(2 n+1) & +v(4 n+5)]+\left[P+L_{2}^{\prime}(n-1)\right]^{2}(u+v+2 n v) \\
& -B P\left[u\left(-n^{2}+2 n+2\right)+v\left(-n^{2}+4 n+6\right)\right] \\
& -2 B L_{2}^{\prime}\left(n^{2}-1\right)(u+3 v) .
\end{aligned}
$$

The coefficients for $i>4$ can be obtained from the recurrence relation 


$$
c_{i}=-c_{i-2} \frac{(\mathrm{i}-2)[u+(i-3) v+2(i-3) n v]\left[B-L_{2}^{\prime}(n-1)-P\right]^{2}}{2 i n[u+(i-1) v](n-1)^{2}},
$$

from which any higher-order coefficient can be obtained. For example, the sixth- and eighth-order coefficients are

$$
\begin{gathered}
c_{6}=-\frac{P(u+3 v+6 n v)\left[B-L_{2}^{\prime}(n-1)-P\right]^{2}}{24 n^{2}(u+3 v)(u+5 v)(n-1)^{5}} \Delta, \\
c_{8}=\frac{P(u+3 v+6 n v)(u+5 v+10 n v)\left[B-L_{2}^{\prime}(n-1)-P\right]^{4}}{64 n^{3}(u+3 v)(u+5 v)(u+7 v)(n-1)^{7}} \Delta .
\end{gathered}
$$

The term $c_{2}$ gives the curvature of the second surface at $x=0$. Indeed, this result for $c_{2}$ is nothing but the lens having power $P$. The term $c_{4}$ provides the second degree of freedom after the base curve. We call this term "fourth-order asphericity" of the surface; it is related with the conic constant when the surface is a conicoid. The terms $c_{6}, c_{8}$, etc., are higher-order asphericity terms.

Let us check the accuracy of the presented approach when the back surface contains terms up to order four. Once we choose values for $P, B, n$, and $L_{2}^{\prime}$, we can compute the coefficients $c_{2}$ and $c_{4}$ by means of (26) and (27). With these coefficients, condition (22) should be satisfied within the approximations used to obtain (18) and (19) and within the fourth-order approximation for the back surface.

The results are compared with those of a numerical computation performed as follows. A computer model of the lens is constructed using the values $P, B, n$, and the thickness that would be used by default in actual lens manufacturing. A fourth-order coefficient $c_{4}=0$ is chosen as seed. The oblique powers are computed by exact ray-tracing through the lens assuming an inverse distance to the rotation center of the eye $L_{2}^{\prime}[6,22]$. The tracing is repeated in an optimization loop that searches the value of $c_{4}$ for which condition (22) is better fulfilled.

The comparison is presented in Figure 5 as curves of tangential (blue) and sagittal (red) power vs the transverse coordinate. Lens data are $P=5 \mathrm{D}, B=6 \mathrm{D}, n=1.5, L_{2}^{\prime}=37$ $\mathrm{D}$, and the optimization was repeated for nine values of $u$ ranging from $-\sqrt{2} / 2$ to $+\sqrt{2} / 2$. Solid lines represent the analytical predictions (up to order four), and the dashed lines represent the optimal solution obtained by numerical methods.

The first conclusion we can draw from Figure 5 is that oblique powers do not behave as expected at full field, that is, for all values of the transverse coordinate used in the computation, up to $15 \mathrm{~mm}$ (which corresponds to $\theta_{2}^{\prime}=29 \mathrm{deg}$ ). This is especially easy to spot for the classical solutions, which occupy the main diagonal of the grid in Figure 5. For punctual lenses $(u=-0.707)$, we expect that $F_{T}=F_{S}$. For Percival lenses $(u=0.707)$, $F_{T}-P$ should equal $P-F_{S}$. For lenses with zero tangential error $(u=0), F_{T}$ should equal $P$. We indeed observe these behaviors but only for values of $x$ smaller than around $5 \mathrm{~mm}$ $\left(\theta_{2}^{\prime}=10 \mathrm{deg}\right)$. As the transverse coordinate grows larger, the error in meeting condition (22) also grows, more or less uniformly across the different values of the parameter $u$. The reason for this error is explained next: When the expressions for the oblique powers are inserted into the condition (22), and we solve for the coefficients of the Taylor expansion of $z(x)$, we find a condition for $c_{2}$ that gives (26), a special relation between $c_{2}$ and $c_{4}$ that gives (27), and the recurrence relation (28) linking $c_{i}$ with $c_{i-2}$ starting with $i=6$.

Let us assume that there are just two terms (second- and fourth-orders) in the series expansion for $z(x)$. The outline procedure then gives two contradictory equations for $c_{4}$, equation (27) and $c_{4}=0$, the latter from the recurrence relation (28) with $c_{6}=0$. The meaning of this is that there is no surface defined with just a quadratic and a quartic coefficient that allows (22) to be fulfilled for all $x$, at least with the approximations used to reach (18) and (19) and with the approximation giving rise to the sagittal and tangential refractive powers of the surface. 

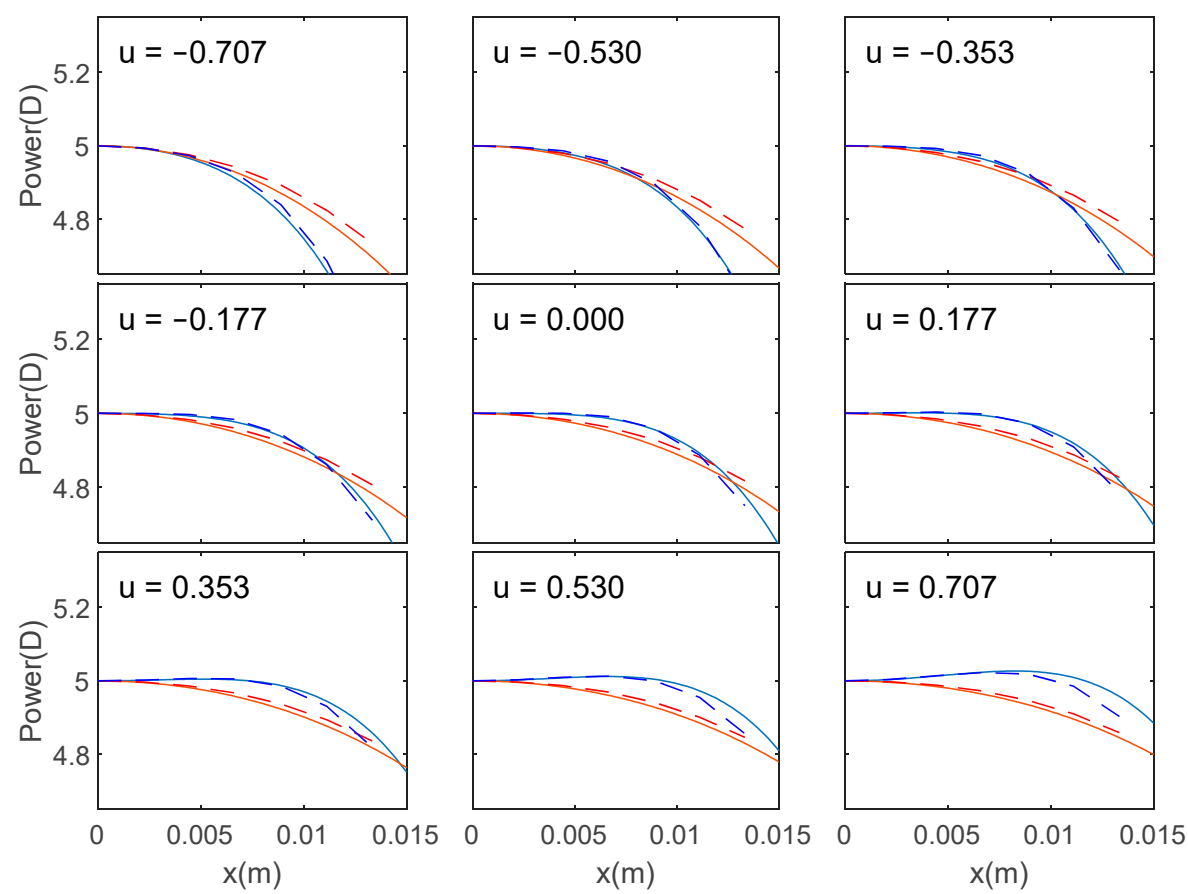

Figure 5. Tangential (blue line) and sagittal (red line) powers up to term $c_{4}$ for a lens with $P=5 \mathrm{D}$, $B=6 \mathrm{D}, n=1.5$, and $L_{2}^{\prime}=37 \mathrm{D}$, for $u$ ranging from -0.707 to +0.707 . The continuous line represents the analytical solution obtained within the approximation described in the text, while the dashed line corresponds to the numerical solution obtained by optimization.

The impossibility of coping with (22) is especially clear for $u=0$. In this case, the tangential power should be constant and equal to $P$. However, Equations (26) and (27) yield

$$
\begin{aligned}
F_{T}(x)=P-x^{4} & \frac{P(2 n+1)\left[B-L_{2}^{\prime}(n-1)-P\right]^{2}}{4 n^{2}(n-1)^{4}}\left(4 B^{2} n+5 B^{2}-6 B L_{2}^{\prime} n^{2}\right. \\
& +6 B L_{2}^{\prime}+B P n^{2}-4 B P n-6 B P+2 L_{2}^{\prime 2} n^{3}-3 L_{2}^{\prime 2} n^{2}+L_{2}^{\prime 2} \\
& \left.+4 L_{2}^{\prime} P n^{2}-2 L_{2}^{\prime} P n-2 L_{2}^{\prime} P+2 P^{2} n+P^{2}\right),
\end{aligned}
$$

and the existence of a non-zero fourth-order term implies that the tangential error cannot be zeroed for all $x$.

In the classical third-order theory, fourth-order terms are accounted for in the surface powers, but only second-order terms are retained in the oblique powers before testing the conditions for oblique astigmatism, power error, or tangential error $[2,8,18]$. The result is that, within the frameworks of these third-order theories, the resulting lenses cope with the zeroing of the selected oblique error at full field. However, this is not how the lens will actually work. In particular, the exact oblique powers of the lens with the optimal $c_{4}$, determined by exact ray tracing, present a remarkably similar behavior as those obtained with the analytic tools presented so far, as shown in Figure 5.

Let us go back to the inconsistency for $c_{4}$ discussed before. If we add a third term with coefficient $c_{6}$, this will be given in terms of $c_{4}$ (which in turn is given in terms of $c_{2}$ ), and the last application of the recurrence formula will give $c_{6}=0$. The inconsistency has been translated to $c_{6}$. In general, for any given number of terms, there will be an inconsistency for the last one. However, we can expect that each high-order coefficient will be much smaller than the previous one. If that is so, condition (22) should be better fulfilled by using more terms in the power expansion for $z(x)$.

We tested this hypothesis with the same lens parameters used in the previous experiment but using coefficients up to $c_{8}$. For the numerical computation, we used a polynomial surface with the same degree, and we optimized the fourth-order asphericity and the two high-order coefficients to minimize the error in condition (22). The results are shown in 
Figure 6, using the same color and line type schemes as before. Once again, the match between the analytical results and the numerical computation is remarkably good up to $x \cong 12 \mathrm{~mm}\left(\theta_{2}^{\prime}=24 \mathrm{deg}\right)$. Additionally, the two techniques now provide a much better fulfillment of condition (22) for any $u$. We observe that the analytical solutions start deviating from the numerical one for $x>12 \mathrm{~mm}$, especially the tangential power. We suspect that at this value of the transverse coordinate, the approximations made on the angles and the curvatures start showing up. The results presented in Figure 6 strongly suggest that higher-order polynomial terms are indeed needed for a full-field correction of the oblique aberrations, even when the oblique powers have been obtained with the low-angle, low-curvature, and negligible-thickness approximations.
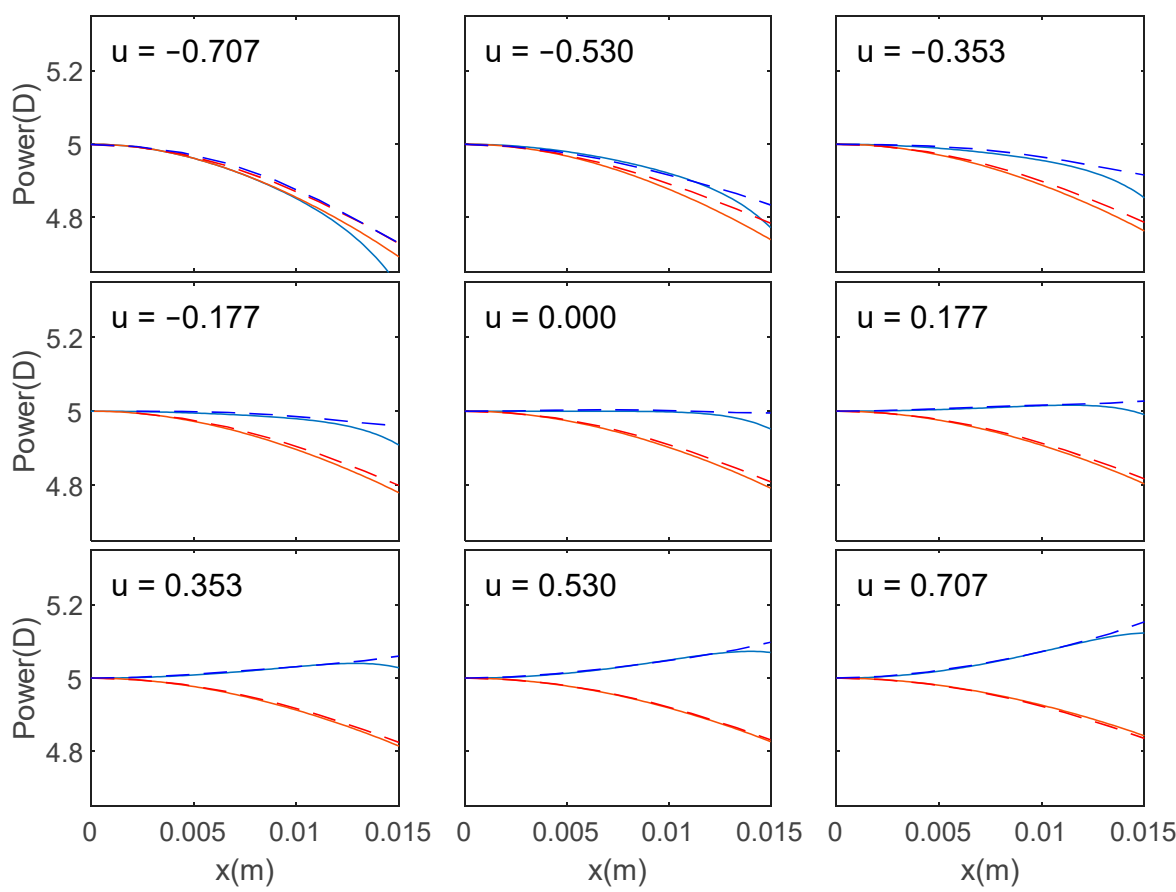

Figure 6. Tangential (blue line) and sagittal (red line) powers up to the term $c_{8}$ for a lens with $P=5 \mathrm{D}$, $B=6 \mathrm{D}, n=1.5$, and $L_{2}^{\prime}=37 \mathrm{D}$, for $u$ ranging from -0.707 to +0.707 . The continuous line represents the analytical solution obtained within the approximation described in the text, while the dashed line corresponds to the exact numerical solution.

It is worth noting that all the coefficients $c_{i}$ with $i>2$ are proportional to $\Delta$. If the lens parameters combine in a way that $\Delta=0$, all these coefficients will be zero, and condition (22) will be satisfied at full field. A surface with a single second-order term is, within the approximations used, nothing but a spherical surface, and the condition $\Delta=0$ expresses the relation between the base curve and the lens power that will satisfy condition (22). $\Delta(u)=0$ is then the equation of the Tscherning ellipse for each value of the parameter $u$.

There is a final consideration about the oblique powers shown in Figure 6. Their behavior is smoother and more "parabolic" than those in Figure 5, where the curves clearly deviate from the parabolic shape when the fourth-order term dominates. This behavior is not a special feature of the chosen example; we have always found the same pattern in many other cases numerically analyzed.

To have a better understanding of this feature, let us assume we start with a back surface defined by a polynomial having $N$ terms and maximum order $2 N$. Using Equations (26)-(29) we find the coefficients $\left\{c_{i}\right\}_{i=2, \ldots, 2 N}$ for which the lens satisfies condition (22). Using these coefficients, we use expressions (18) and (19) to find the polynomial expansions for the oblique powers. We can easily check that these polynomials have the same order than $z(x)$, so $F_{T}=P+\sum_{i=2,4, \ldots, 2 N} c_{T i} x^{i}$ and $F_{S}=P+\sum_{i=2,4, \ldots, 2 N} c_{S i} x^{i}$. 
Although we have not found analytical proof, the numerical analysis of many cases suggests that, for an $N$ that is large, the next property holds:

$$
\sum_{i=4,6, \cdots, 2 N} c_{T i} x^{i} \ll P+c_{T 2} x^{2}, \sum_{i=4,6, \cdots, 2 N} c_{S i} x^{i} \ll P+c_{S 2} x^{2} .
$$

In other words, the more terms we use in $z(x)$, the better condition (22) is fulfilled for all $x$, and the smaller is the sum of all the higher-than-fourth-order terms in the series that give the oblique powers. This property is illustrated in Figure 7, where we show the tangential power corresponding to the example analyzed in Figure 6, using $F_{T}=P+c_{T 2} x^{2}$ (blue curve), and the complete $F_{T}=P+\sum_{i=2,4, \cdots, 8} c_{T i} x^{i}$ (orange curve). The maximum difference between the two curves at $x=10 \mathrm{~mm}$ is about $0.01 \mathrm{D}$. This property is closely related with the use of solutions of condition (22) as optimizers of more general merit functions involving blur.

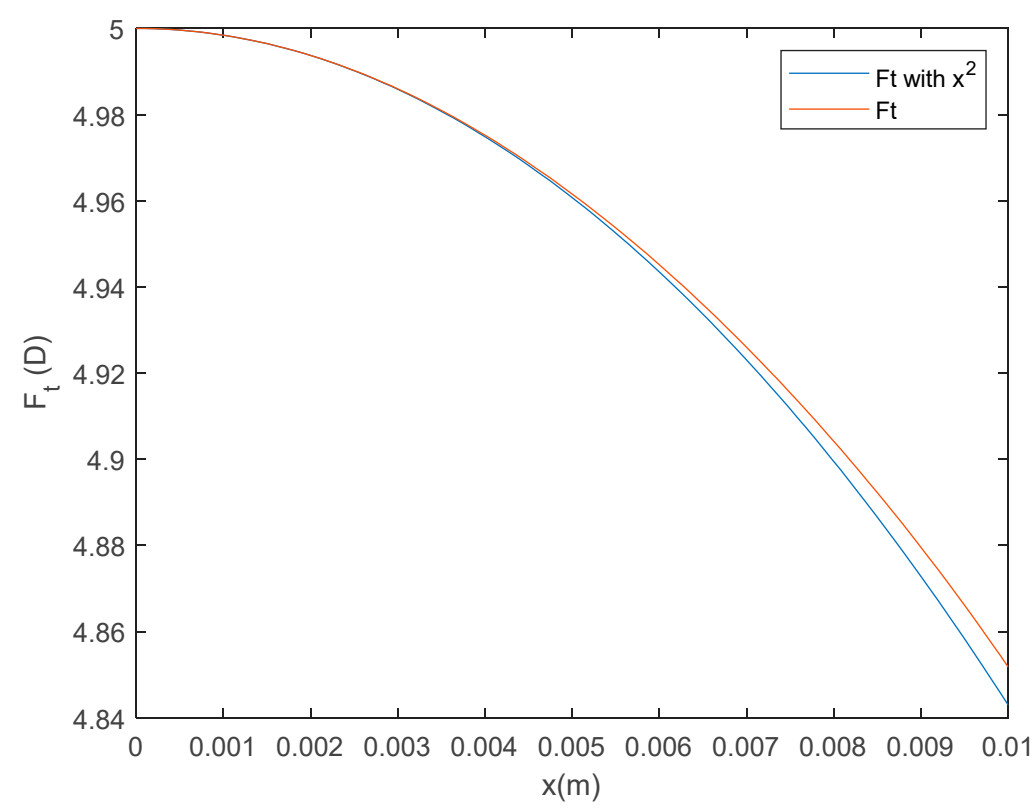

Figure 7. Tangential power for a lens with $P=5 \mathrm{D}, B=6 \mathrm{D}, n=1.5$, and $L_{2}^{\prime}=37 \mathrm{D}$, computed for $u=-0707$. It was computed from Equation (18) using an eighth-order expansion for the back surface. The orange line is the tangential power computed with all the terms resulting from (18), while the blue curve has been computed only with the quadratic term.

\section{Discussion}

Several authors have pointed out that the fourth-order asphericity is well defined for any pair $(B, P)$ and for the three classical solutions $[3,9,12]$. In other words, in classical third-order theory, for any pair $(B, P)$ we can always find a coefficient $c_{4}$ for which the lens meets one of the three classical conditions $E_{A}=0, E_{P}=0$, or $E_{T}=0$.

According to the theory presented in this paper, the fourth-order asphericity is well defined except when $u+3 v=0$, that is, when $u=-3 v$. By inspection of the recurrence relation (29) and examples (30) and (31), we can see that any higher-order term $c_{i}$ fails to be well defined for all $u_{j}$, such that $\left\{u_{j}=-(j-1) v\right\}_{j=4,6, \cdots, i}$.

If we use the normalization condition (23), we can state that any theory of order $2 \mathrm{~N}$, that is, a theory using monomials up to order $2 N$ in the expansion of $z(x)$, will fail to meet condition (22) for the set of values of the parameter $u$ satisfying

$$
u_{j}=\frac{1-j}{\sqrt{1+(1-j)^{2}}}, j=4,6, \cdots, 2 \mathrm{~N}
$$


This is a decreasing monotonic sequence with limit -1 and starting at $u_{4}=3 / \sqrt{10}$. We conclude that the general problem stated by condition (22) has a solution for any pair $(P, B)$ when $u \in(-3 / \sqrt{10}, 1]$. This interval includes the classical solutions: point focal lenses, Percival lenses, and lenses with zero tangential error. Lenses with zero sagittal error $(u=-1)$ or with very small sagittal error $(u \gtrsim-1)$ lie in the complementary interval $u \in[-1,-3 / \sqrt{10}]$. In this interval, and whenever Equation (36) is satisfied for a given $j$, all the coefficients with $i>j$ are not defined. Lenses with identically zero sagittal error can be computed, as all the coefficients are finite. However, convergence of expansions (25) will be slower than for those problems with bigger values of $u$.

Despite the fact that Equations (27)-(30) are not linear on $u$, we can find the relationships between the asphericity parameters of a given order corresponding to different values of $u$. For example, let us call $c_{4 p}, c_{4 m}$, and $c_{4 t}$ to the fourth-order asphericities corresponding to point-focal lenses, Percival lenses, or zero tangential error lenses, respectively. It can be shown that

$$
c_{4 t}=\frac{2}{3} c_{4 m}+\frac{1}{3} c_{4 p},
$$

that is, the fourth-order asphericity coefficient of the lens with zero tangential error is a weighted sum of the coefficients for the Percival lens and the point-focal lens. Among the classical solutions, asphericity for zero tangential errors provides a more balanced solution.

Figure 8 shows the curve $c_{4}(u)$ in the interval $u=(-\sqrt{3} / 10,1]$ for the example lens used in Figures 5-7. The amount of asphericity needed to meet some design criterion, whether it is a determined value of $u$ or the use of other merit functions, which are related, as we show next, is of great value to the lens designer. In general, smaller values of asphericity may facilitate the lens manufacturing and will make the lens more stable with respect to variation of the wearer's parameters or lens misalignment.

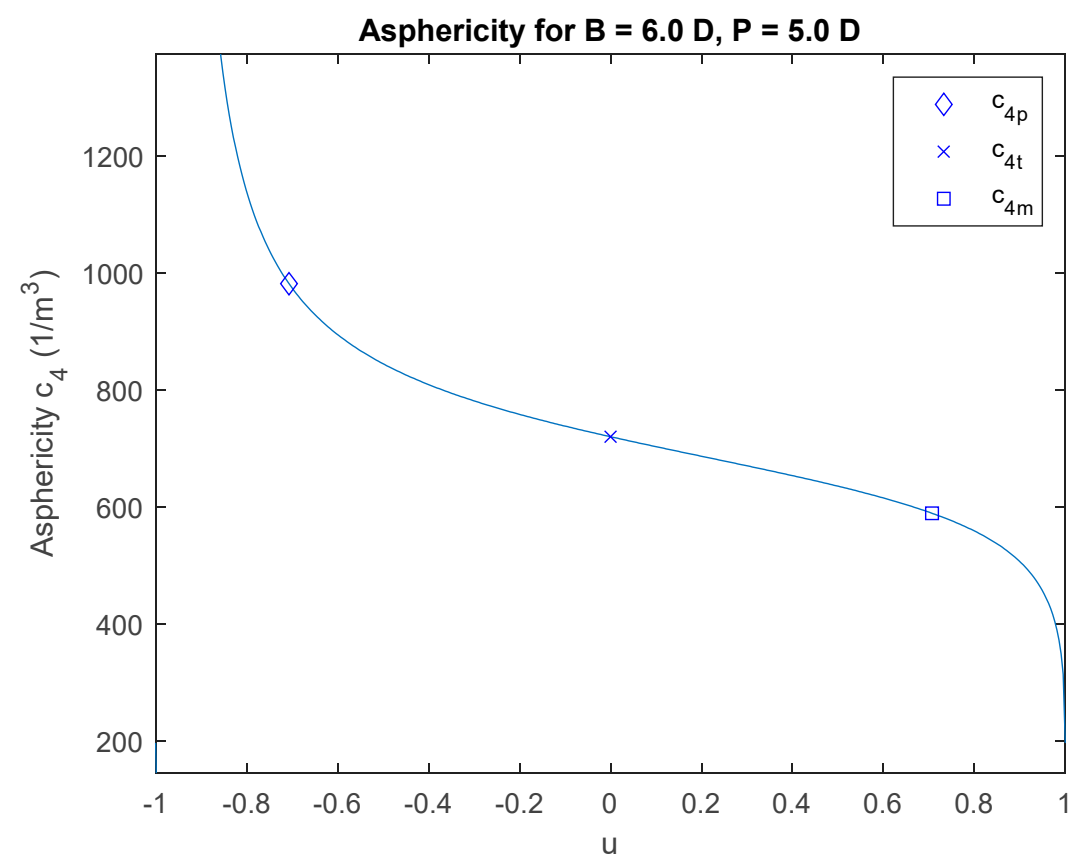

Figure 8. Fourth-order asphericity $c_{4}$ as a function of $u$ for the same lens example used in Figures 5-7.

We finally discuss the relation between condition (22) and the use of more general merit functions that may balance combinations of the oblique powers across the complete field of view. A standard example of such merit function can be

$$
f=\sum_{k} w_{k}\left[\left(\alpha P_{T}\left(x_{k}\right)+\beta P_{S}\left(x_{k}\right)-P_{t g t}\left(x_{k}\right)\right]^{2}\right.
$$


where $\alpha$ and $\beta$ provides a weighted average of the oblique powers that are evaluated at points $x_{k}$, as it is the target power $P_{t g t}\left(x_{k}\right)$, and $W_{k}$ are weights controlling the importance given to the transverse coordinate.

The idea behind this approach is that the surface minimizing (36) can be found among the surfaces that comply with (22) and whose coefficients are given by (26) to (29). If that were the case, we would have converted a multi-parametric optimization of the surface coefficients into the minimization of a functional that depends on a single variable.

The merit functions are usually constructed to balance the error between sagittal and tangential power or between mean power and astigmatism. One instance of these merit functions consists of the minimization of the defocus defined by Raasch [19], which is given by

$$
\delta(x)=\sqrt{\left[F_{S}(x)-P\right]^{2}+\left[F_{T}(x)-P\right]^{2}}
$$

For example, we could choose

$$
f=\int_{0}^{\hat{x}} \delta^{2}(x) d x,
$$

where $\hat{x}$ is a limiting value for the field of view. Another example is the "rational lens" [12].

Let us consider a more general merit function of the form:

$$
\begin{gathered}
f=\int_{0}^{\hat{x}}\left\{w_{1}\left[F_{S}(x)-P\right]^{2}+w_{2}\left[F_{T}(x)-P\right]^{2}+w_{3}\left[F_{S}(x)+F_{T}(x)-2 P\right]^{2}\right. \\
\left.+w_{4}\left[F_{S}(x)-F_{T}(x)\right]^{2}\right\} d x
\end{gathered}
$$

which aims to reduce tangential error, sagittal error, power error, and astigmatism uniformly over the transverse coordinate and with weights that can be organized in a single 4-tupla $w=\left(w_{1}, w_{2}, w_{3}, w_{4}\right)$. This general merit function includes the minimization of the Raasch defocus when the weight vector is set to $w=(1,1,0,0)$.

Let $z_{u}(x)$ be the surfaces that meet condition (22) for the parameter $u$, and let us try to minimize $f$ in the set of functions $\left\{z_{u}\right\}$. Now, $f$ depends on the single variable $u$ (which will determine the coefficients $\left.\left\{c_{i}\right\}_{i=2, \cdots, 2 N}\right)$, and we just must impose $\partial f / \partial u=0$. The problem can be analytically solved if $N$ is large enough so that property (33) holds. In that case we use $F_{T}=P+c_{T 2} x^{2}$ and $F_{S}=P+c_{S 2} x^{2}$, where the coefficients are given by

$$
\begin{gathered}
c_{T 2}=\frac{L_{2}^{\prime 2} P u}{u+3 v}+\frac{P u\left(B^{2}-L_{2}^{\prime 2}+2 L_{2}^{\prime} P-P^{2}\right)}{n(u+3 v)}-\frac{P u\left(B^{2}+B P-P^{2}\right)}{(u+3 v)(n-1)}, \\
c_{S 2}=-\frac{L_{2}^{\prime 2} P v}{u+3 v}-\frac{P v\left(B^{2}-L_{2}^{\prime 2}+2 L_{2}^{\prime} P-P^{2}\right)}{n(u+3 v)}+\frac{P v\left(B^{2}+B P-P^{2}\right)}{(u+3 v)(n-1)}
\end{gathered}
$$

Now, the minimization of (39) can be analytically obtained, with the result

$$
u_{\text {min }}=\frac{w_{1}+4 w_{3}-2 w_{4}}{\sqrt{w_{1}^{2}+8 w_{1} w_{3}-4 w_{1} w_{4}+9 w_{2}^{2}+24 w_{2} w_{3}+12 w_{2} w_{4}+32 w_{3}^{2}+8 w_{4}^{2}}} .
$$

For the particular case of the Raasch defocus, the optimum value for $u$ is $u_{\min }=$ $1 / \sqrt{10} \cong 0.316$. This prediction has been validated with the result obtained when the Raasch defocus is numerically minimized with exact ray tracing and multi-parametric optimization of the surface coefficients. Figure 9 shows the tangential and sagittal powers for the lens with parameters $P=-4 \mathrm{D}, B=0.5 \mathrm{D}, n=1.5$, and $L_{2}^{\prime}=37 \mathrm{D}$, which satisfies condition (22) with $u=0.316$, compared with the oblique powers of the lens numerically optimized. The difference between the two approaches is below $0.01 \mathrm{D}$ for the range of transverse coordinates for which the angles and curvature approximations make sense. The prediction fits the numerical result quite well, which indicates that the previous method is 
adequate for exploring lens performance using nonlinear merit functions without the need for numerical optimization.

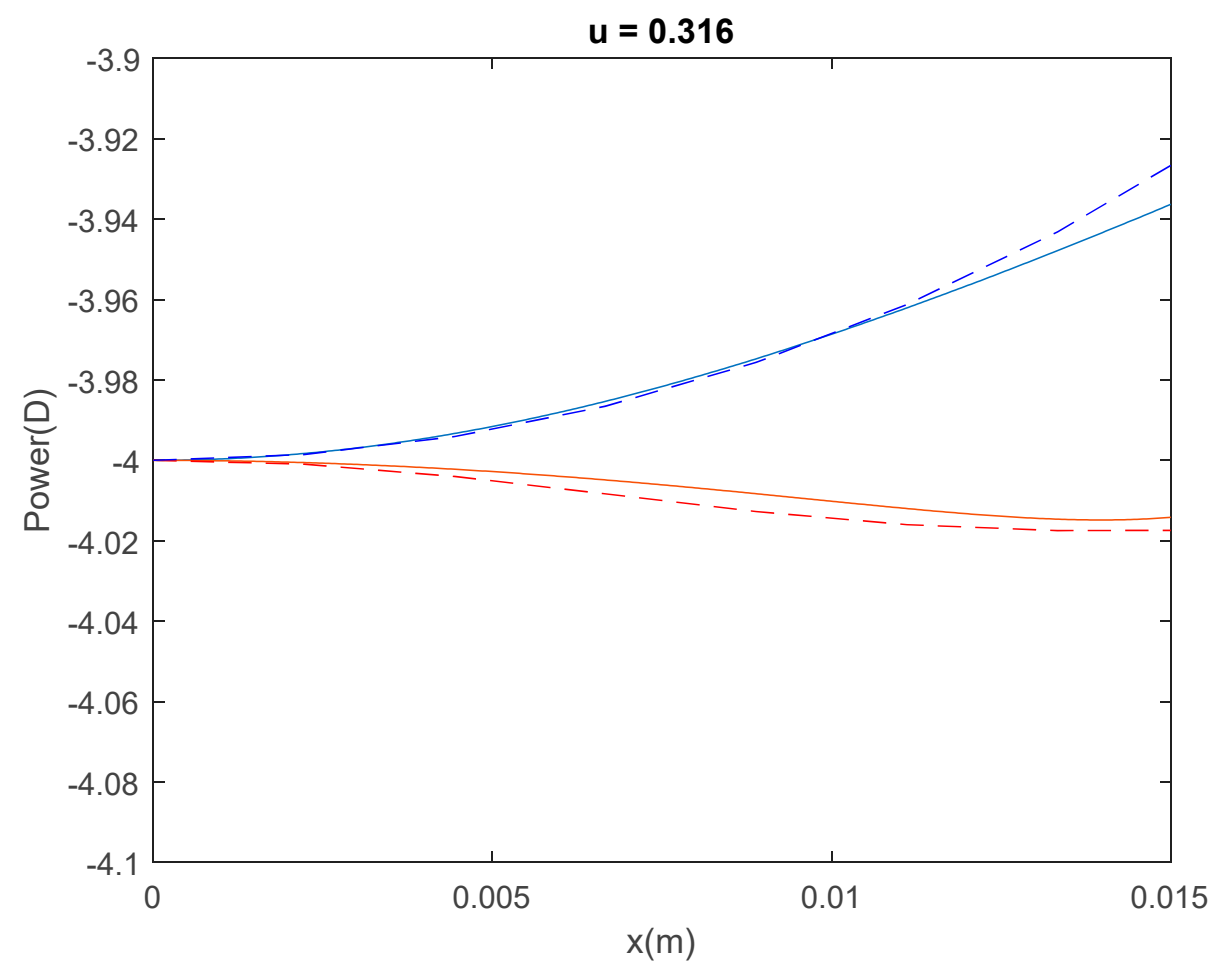

Figure 9. Tangential (red line) and sagittal (blue line) powers up to the term $c_{8}$ for a lens with $P=-4 \mathrm{D}, B=0.5 \mathrm{D}, n=1.5$, and $L_{2}^{\prime}=37 \mathrm{D}$. The continuous lines represent the analytical solution obtained with the approximation described in the text for $u=0.316$, while the dashed lines correspond to the oblique power of the lens computed by multiparametric minimization of the merit function (39) with weights $(1,1,0,0)$.

\section{Conclusions}

This work shows that it is possible to use high-order polynomial terms to describe the optical surface of an ophthalmic lens while at the same time maintaining the second-order approximation of the incident and refraction angles typically used in the so-called thirdorder theory of spectacle lenses. There is previous work that used high-order aspherical terms to correct oblique aberrations in ophthalmic lenses [1,17], but the calculation has always been based on multiparametric optimization. Here, we presented analytical expressions for the surface coefficients $c_{2}$ up to $c_{8}$ and a general recursive relation for higher-order terms. The need for higher-order polynomial terms in the surface description may seem to contradict the second-order approximation in angles shown in Equation (9), but in fact, both are independent: high-order asphericity may bring richness to the central region of the lens where the incident angles are still small, as demonstrated by the numerical results and corroborated by the analytical approach.

We verified that this formulation provides a more accurate estimation of the oblique powers than previous third-order approximations because they closely match the equivalent ray-traced solutions.

A generalization of the three classical solutions (point-focal lens, Percival lens, and lens with zero tangential error) was proposed, depending on a parameter $u$. We have shown that these generalized solutions are well-defined for all base curves and powers for a wide range of values of the parameter $u$. Moreover, we have shown that the oblique powers of the solutions obtained with this parametrization tend to be parabolic and smooth. Moreover, it seems that the surfaces that minimize a broad set of nonlinear merit functions 
belong to the set of functions that meets the general compromise (22). This hypothesis allows the analytical solving of minimization problems relevant in ophthalmic lenses.

Though modern ophthalmic lens design will rely on numerical computation to optimize general lenses with tilts, prescription astigmatism, even progressive lenses, the tools presented in this paper offer new insight on the behavior of the oblique powers and their relationship with the surface geometry and allow fast testing of new design hypotheses.

Author Contributions: Conceptualization, E.P.; methodology, E.P., J.A.G.-P. and J.A.; software, E.P.; validation, E.P., J.A.G.-P. and J.A.; formal analysis, E.P.; investigation, E.P., J.A.G.-P. and J.A.; resources, J.A.G.-P. and J.A.; data curation, J.A.G.-P. and J.A.; writing-original draft preparation, E.P.; writingreview and editing, J.A.G.-P. and J.A.; visualization, E.P., J.A.G.-P. and J.A.; supervision, J.A.G.-P. and J.A.; project administration, E.P., J.A.G.-P. and J.A.; funding acquisition, J.A.G.-P. and J.A. All authors have read and agreed to the published version of the manuscript.

Funding: This work was partially financed by the European Fund for Regional Development (EFRDFEDER) and the Spanish Agencia Estatal de Investigación, through the grant DPI2016-75272-R.

Institutional Review Board Statement: Not applicable.

Informed Consent Statement: Not applicable.

Data Availability Statement: Data sharing not applicable.

Conflicts of Interest: The authors declare no conflict of interest.

\section{References}

1. Sun, W.-S. Ophthalmic lens design with the optimization of the aspherical coefficients. Opt. Eng. 2000, 39, 978. [CrossRef]

2. Smith, G.; Atchison, D.A. Effect of Conicoid Asphericity on the Tscherning Ellipses of Ophthalmic Spectacle Lenses. J. Opt. Soc. Am. 1983, 73, 441-445. [CrossRef] [PubMed]

3. Atchison, D.A. Third-order theory and aspheric spectacle lens design. Ophthalmic Physiol. Opt. 1984, 4, 179-186. [CrossRef] [PubMed]

4. Jalie, M. Modern spectacle lens design. Clin. Exp. Optom. 2020, 103, 3-10. [CrossRef] [PubMed]

5. Landgrave, J.E.A.; Moya-Cessa, J.R. Generalized Coddington equations in ophthalmic lens design. J. Opt. Soc. Am. A 1996, 13, 1637. [CrossRef]

6. Jalie, M. The Principles of Ophtalmic Lenses, 4th ed.; Association of British Dispensing Opticians: London, UK, 1992.

7. Atchison, D.A.; Smith, G. Spectacle Lenses and Third-Order Distortion. Ophthalmic Physiol. Opt. 1987, 7, 303-308. [CrossRef] [PubMed]

8. Miks, A.; Novak, J.; Novak, P. Third-order design of aspheric spectacle lenses. Optik 2010, 121, 2097-2104. [CrossRef]

9. Atchison, D.A. Spectacle lens design: A review. Appl. Opt. 1992, 31, 3579. [CrossRef] [PubMed]

10. Davis, J.K.; Fernald, H.G.; Rayner, A.W. The design of a general purpose single vision lens series. Optom. Vis. Sci. 1965, 42, 203-236. [CrossRef] [PubMed]

11. Von Rohr, M.; Würdigung, Z.; von Scheiners, A. Archiv für Augenheilkunde; Springer: Berlin/Heidelberg, Germany, 1920; Volume 86, pp. 247-263.

12. Le Texier, F.; Lenne, W.; MERCIER, J.L. Generalization of the Tscherning Theory: Optimization of Aspheric Ophthalmic Lenses. Ophthalmic Physiol. Opt. 1987, 7, 63-72. [CrossRef] [PubMed]

13. Rojo, P.; Royo, S.; Ramírez, J.; Madariaga, I. Numerical implementation of generalized Coddington equations for ophthalmic lens design. J. Mod. Opt. 2014, 61, 204-214. [CrossRef]

14. Atchison, D.A. Modern optical design assessment and spectacle lenses. Opt. Acta Int. J. Opt. 1985, 32, 607-634. [CrossRef]

15. Smith, G.; Atchison, D.A. Aspheric Surfaces and Lenses in Ophthalmic Optics. Clin. Exp. Optom. 1985, 68, 125-132. [CrossRef]

16. Barbero, S. Minimum tangential error ophthalmic lens design without multi-parametric optimization. Opt. Commun. 2012, 285, 2769-2773. [CrossRef]

17. Katz, M. Aspherical surfaces used to minimize oblique astigmatic error, power error, and distortion of some high positive and negative power ophthalmic lenses. Appl. Opt. 1982, 21, 4399_1. [CrossRef] [PubMed]

18. Smith, G.; Atchison, D.A. Construction, specification, and mathematical description of aspheric surfaces. Optom. Vis. Sci. 1983, 60, 216-223. [CrossRef] [PubMed]

19. Raasch, T.W. Spherocylindrical refractive errors and visual acuity. Optom. Vis. Sci. 1995, 72, 272-275. [CrossRef] [PubMed]

20. Kempen, J.H. The Prevalence of Refractive Errors among Adults in the United States, Western Europe, and Australia. Arch. Ophthalmol. 2004, 122, 495-505. [PubMed]

21. Liu, J.; Miñano, J.C.; Benítez, P. Double aspheric imaging design with unconstrained object to image mapping. Opt. Express. 2015, 23, 13370. [CrossRef] [PubMed]

22. Alonso, J.; Gómez-Pedrero, J.A.; Quiroga, J.A. Modern Ophthalmic Optics; Cambridge University Press: Cambridge, UK, 2019. 